\title{
Phenothiazines induce PP2A-mediated apoptosis in T cell acute lymphoblastic leukemia
}

\begin{abstract}
Alejandro Gutierrez, 1,2 Li Pan, ${ }^{3}$ Richard W.J. Groen, ${ }^{4,5}$ Frederic Baleydier, ${ }^{3,6}$ Alex Kentsis, ${ }^{1,2}$ Jason Marineau, ${ }^{4}$ Ruta Grebliunaite, ${ }^{1}$ Elena Kozakewich, ${ }^{1}$ Casie Reed, ${ }^{1}$ Francoise Pflumio,7,8 Sandrine Poglio, 7,8 Benjamin Uzan,, 7,8 Paul Clemons, ${ }^{9}$ Lynn VerPlank, ${ }^{9}$ Frank An, ${ }^{9}$ Jason Burbank, ${ }^{9}$ Stephanie Norton, ${ }^{9}$ Nicola Tolliday, ${ }^{9}$ Hanno Steen, ${ }^{10}$ Andrew P. Weng, ${ }^{11}$ Huipin Yuan, 12 James E. Bradner, ${ }^{4}$ Constantine Mitsiades, ${ }^{4}$ A. Thomas Look, ${ }^{1,2}$ and Jon C. Aster ${ }^{3}$

1Department of Pediatric Oncology, Dana-Farber Cancer Institute and Harvard Medical School, Boston, Massachusetts, USA. 2Division of Hematology/Oncology, Boston Children's Hospital and Harvard Medical School, Boston, Massachusetts, USA. ${ }^{3}$ Department of Pathology, Brigham and Women's Hospital and Harvard Medical School, Boston, Massachusetts, USA. ${ }^{4}$ Department of Medical Oncology, Dana-Farber Cancer Institute and Harvard Medical School, Boston, Massachusetts, USA. ${ }^{5}$ Department of Cell Biology, University Medical Center Utrecht, Utrecht, the Netherlands. ${ }^{6}$ Department of Pediatric Hematology, Hospices Civils de Lyon, Lyon, France.

${ }^{7}$ Laboratoire de Recherche sur les Cellules Souches Hématopoïétiques et Leucémiques, Institut de Radiobiologie Cellulaire et Moléculaire, Direction des Sciences du Vivant, Commissariat à l'Energie Atomique et aux Energies Alternatives, Institut National de la Santé et de la Recherche Médicale, Fontenay-aux-Roses and Université Paris-Sud, Fontenay-aux-Roses, France. ${ }^{8}$ Université Paris-Diderot, Paris, France. ${ }^{9}$ Broad Institute, Cambridge, Massachusetts, USA. ${ }^{10}$ Proteomics Center and Department of Pathology, Boston Children's Hospital and Harvard Medical School, Boston, Massachusetts, USA. ${ }^{11}$ British Columbia Cancer Agency, Vancouver, Canada. ${ }^{12}$ Xpand Biotechnology BV, Bilthoven, the Netherlands.
\end{abstract}

T cell acute lymphoblastic leukemia (T-ALL) is an aggressive cancer that is frequently associated with activating mutations in NOTCH1 and dysregulation of MYC. Here, we performed 2 complementary screens to identify FDA-approved drugs and drug-like small molecules with activity against T-ALL. We developed a zebrafish system to screen small molecules for toxic activity toward MYC-overexpressing thymocytes and used a human T-ALL cell line to screen for small molecules that synergize with Notch inhibitors. We identified the antipsychotic drug perphenazine in both screens due to its ability to induce apoptosis in fish, mouse, and human T-ALL cells. Using ligand-affinity chromatography coupled with mass spectrometry, we identified protein phosphatase 2A (PP2A) as a perphenazine target. T-ALL cell lines treated with perphenazine exhibited rapid dephosphorylation of multiple PP2A substrates and subsequent apoptosis. Moreover, shRNA knockdown of specific PP2A subunits attenuated perphenazine activity, indicating that PP2A mediates the drug's antileukemic activity. Finally, human T-ALLs treated with perphenazine exhibited suppressed cell growth and dephosphorylation of PP2A targets in vitro and in vivo. Our findings provide a mechanistic explanation for the recurring identification of phenothiazines as a class of drugs with anticancer effects. Furthermore, these data suggest that pharmacologic PP2A activation in T-ALL and other cancers driven by hyperphosphorylated PP2A substrates has therapeutic potential.

\section{Introduction}

$\mathrm{T}$ cell acute lymphoblastic leukemia (T-ALL) is an aggressive cancer affecting mainly adolescents and young adults. Intensified treatment regimens have improved outcomes, but patients who fail conventional therapy have a dismal prognosis, and T-ALL remains fatal in $20 \%$ of children and more than $50 \%$ of adults (1-3). New therapies are desperately needed for patients in these poor-prognosis groups.

One of the most common genetic aberrations in T-ALL is activating mutations in NOTCH1 (4), a transmembrane receptor that is converted to a transcriptional activator through a series of proteolytic cleavage events, the last of which is carried out by the intramembranous protease $\gamma$-secretase (5). $\gamma$-Secretase cleavage allows the intracellular domain of NOTCH1 (ICN1) to translocate to the nucleus and form a transcriptional activation complex. The

Authorship note: Alejandro Gutierrez, Li Pan, Richard W.J. Groen, Frederic Baleydier and Alex Kentsis contributed equally to this work.

Conflict of interest: The authors have declared that no conflict of interest exists. Citation for this article: J Clin Invest. 2014;124(2):644-655. doi:10.1172/JCI65093. mutations in NOTCH1 that occur in T-ALL variously stimulate NOTCH1 proteolysis and generation of ICN1 or decrease ICN1 turnover, thereby enhancing expression of ICN1 target genes. One of the most important direct targets of ICN1 in the context of T-ALL cells is MYC (6-8); indeed, enforced expression of MYC can rescue some human T-ALL cell lines from NOTCH1 inhibition $(6,9)$ and MYC transgenes can drive T-ALL development in mouse (10) and zebrafish $(11,12)$ models. However, NOTCH1 activation and MYC overexpression are not sufficient for T-ALL development, indicating that establishment and maintenance of T-ALL depends on additional cooperative genetic or epigenetic events that dysregulate other signaling pathways. Indeed, activating NOTCH1 mutations are found together with a diverse collection of other recurrent mutations in human T-ALL (13). Among the most frequent are mutations that activate the PI3K/AKT signaling pathway (14), which has a complex interrelationship with NOTCH signaling in T-ALL cells (15) and is another promising therapeutic target in this disease (16).

Repurposing of FDA-approved drugs is an attractive approach to drug discovery, as it can in principle enable rapid translation to the clinic. Using 2 complementary screens, we identified phenothi- 
A

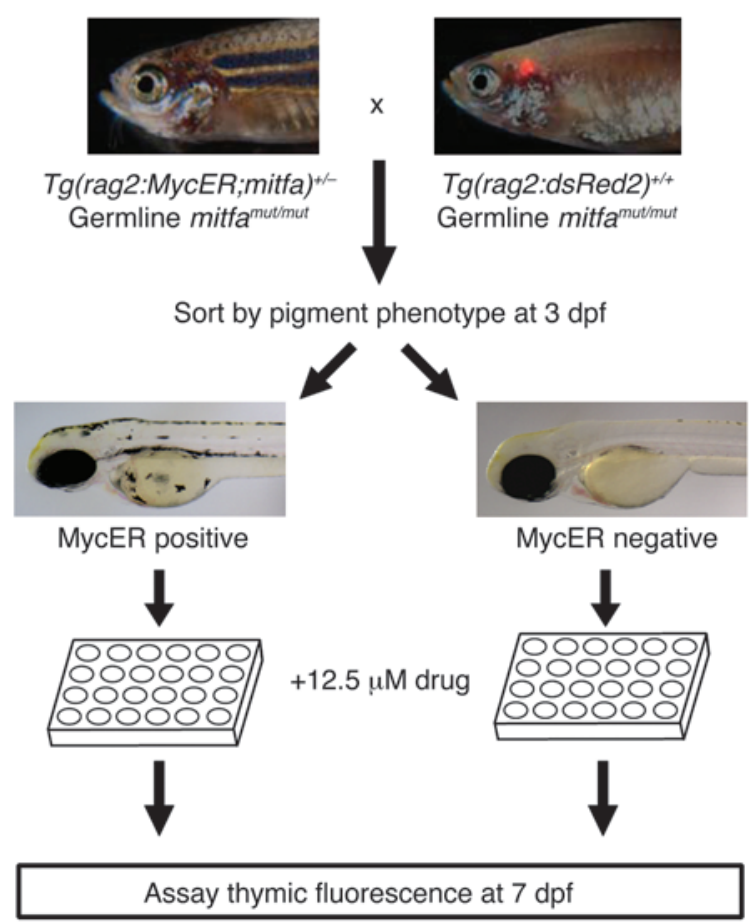

B

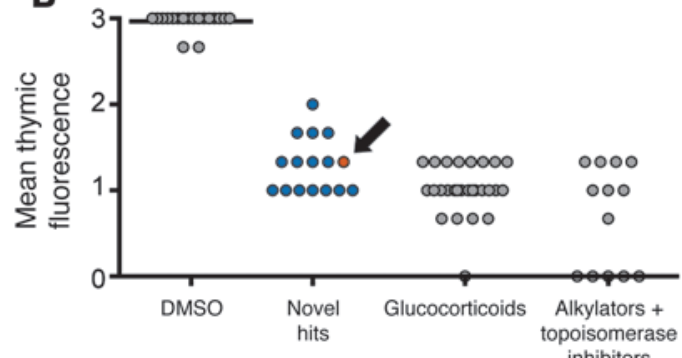

C
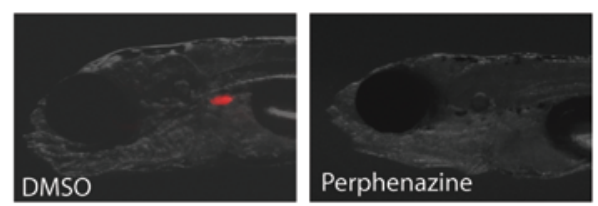

D

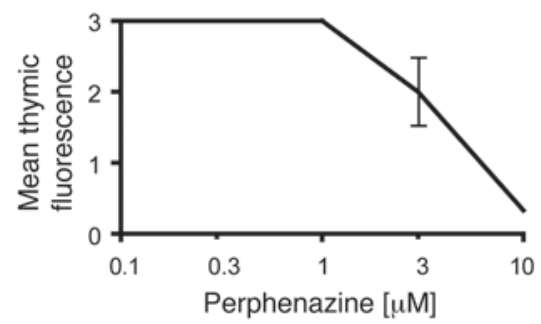

Figure 1

Zebrafish screen for small molecules that are toxic to MYC-overexpressing thymocytes. (A) Primary screen design. (B) Hits from the primary screen. Arrow denotes the result obtained with PPZ (C). Representative images of DMSO (control) or PPZ-treated zebrafish larvae. (D) Doseresponse curve from secondary screen of PPZ, with 6 zebrafish larvae treated per concentration. Drug doses higher than $10 \mu \mathrm{M}$ induced general toxicity (not shown). Error bars = SD.

azines as a class of drugs with NOTCH-independent anti-T-ALL activity. Phenothiazines have been used for over 50 years as neuroleptic-type antipsychotic medications. The antipsychotic effects of phenothiazines correlate with their ability to block dopamine receptors, but a broad array of other activities have been described, including antitumor effects. The basis for the antiproliferative activities of phenothiazines are uncertain and have been variously attributed to a number of mechanisms, including inhibition of PKC (17), calmodulin (18), PI3K/AKT signaling (18-21), and cancer stem cell activity (22).

Using quantitative mass spectrometry to analyze drug-protein binding proteome-wide, we identified the tumor suppressive serine/threonine phosphatase protein phosphatase $2 \mathrm{~A}$ (PP2A) as a new phenothiazine target (Alex Kentsis and James E. Bradner, personal correspondence). Phenothiazines stimulate rapid dephosphorylation of multiple PP2A targets implicated in tumor cell growth and survival in cells, and have antitumor effects on T-ALL cells in vitro and in vivo. Our findings provide a likely explanation for the recurrent discovery of phenothiazines in screens for compounds with anticancer effects, point to new rational drug combinations for consideration in treatment of NOTCH-driven cancers such as T-ALL, and provide additional impetus for development and testing of PP2A activators in a wide variety of cancers.

\section{Results}

Zebrafish screen for small molecules with activity against MYC-overexpressing thymocytes. We previously developed a zebrafish model of MYCinduced T-ALL that closely resembles the human disease morpho- logically and by gene expression $(11,12,23)$, and we wanted to exploit this model for in vivo drug discovery. We thus developed a fluorescence-based screen that was designed to identify small molecules that are selectively cytotoxic to MYC-overexpressing thymocytes. Heterozygous zebrafish carrying a tandem rag2:MYCER;mitfa transgene (created by cointegration of rag2:MYC-ER and wild-type mitfa transgenes at the same genomic locus) were mated to rag2: dsRed2 homozygous animals, generating rag2:MYC-ER-positive or -negative embryos that both express rag2:dsRed2. All crosses were performed in a nacre pigment-mutant background in which melanophores were absent due to homozygous mitfa-inactivating mutations (24). The rescue of the pigmentation defect in fish carrying the rag2:MYC-ER;mitfa transgene allowed us to distinguish MYC-ER-positive and -negative zebrafish at 3 days post-fertilization (dpf) (Figure 1A). At $3 \mathrm{dpf}$, zebrafish larvae were arrayed into 96-well plates (3 larvae/well) and incubated with $12.5 \mu \mathrm{M}$ drug and 4-hydroxytamoxifen to activate the MYC-ER fusion protein. Four days later, microscopy was performed to assay thymic fluorescence, which was scored as 3 (normal), 2 (intermediate), 1 (weak), or 0 (no fluorescence) (Supplemental Figure 1; supplemental material available online with this article; doi:10.1172/JCI65093DS1). Any compound that induced complete loss of fluorescence in 1 fish or weak fluorescence in 2 of the 3 larvae per well, was considered a hit in this primary screen. Compounds that induced either death of larvae or generalized developmental abnormalities were excluded from further analysis. Four libraries of bioactive small molecules comprising 4,880 FDA-approved drugs, drug-like small molecules, and natural products were screened in this fashion. 
A

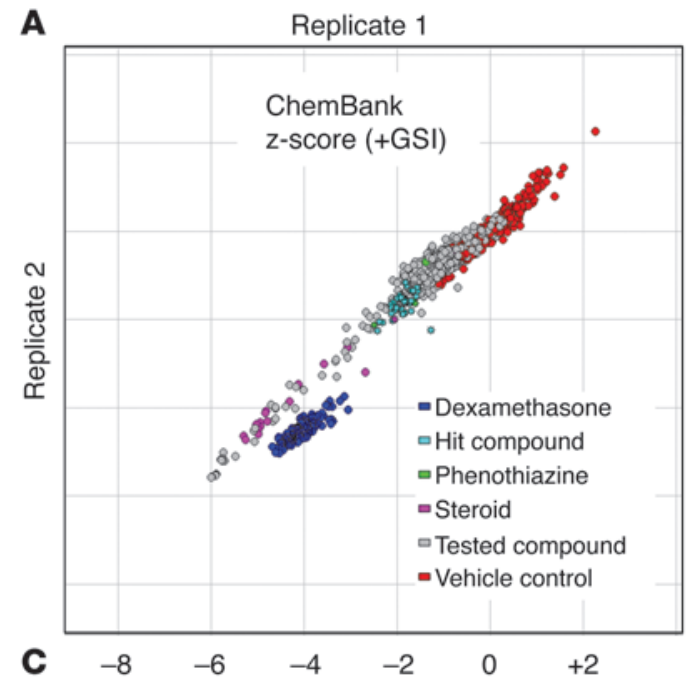

B

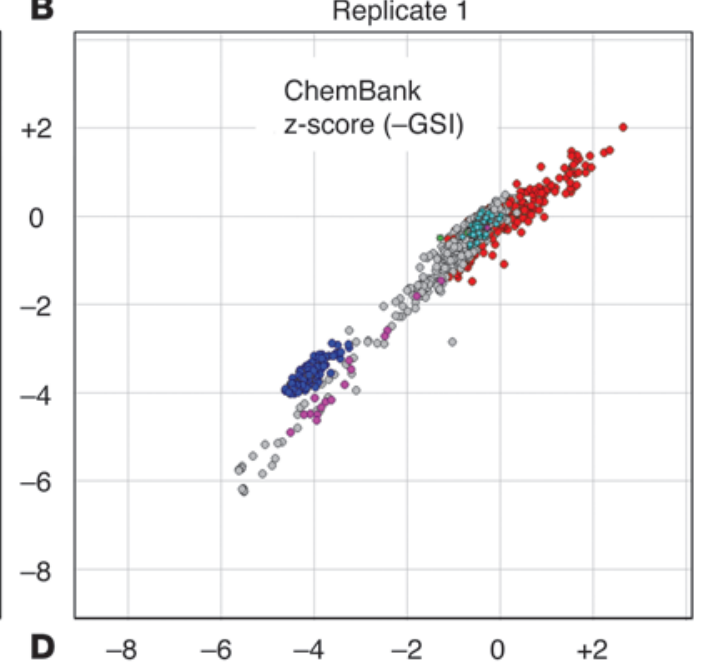

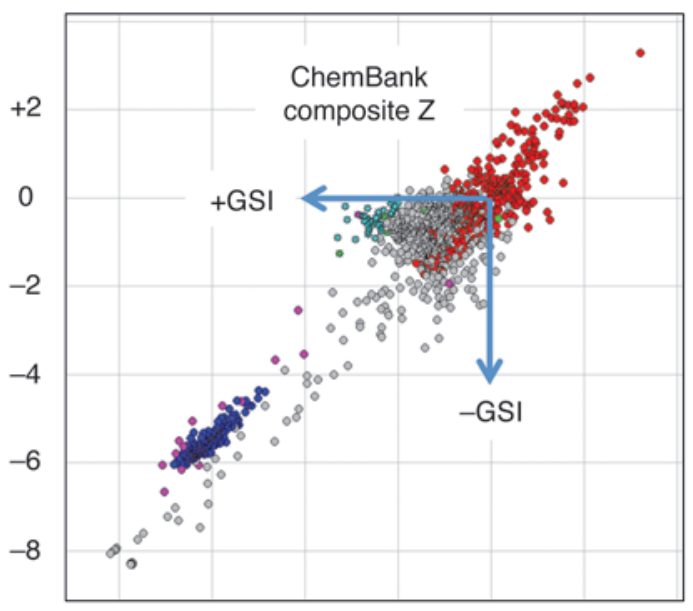

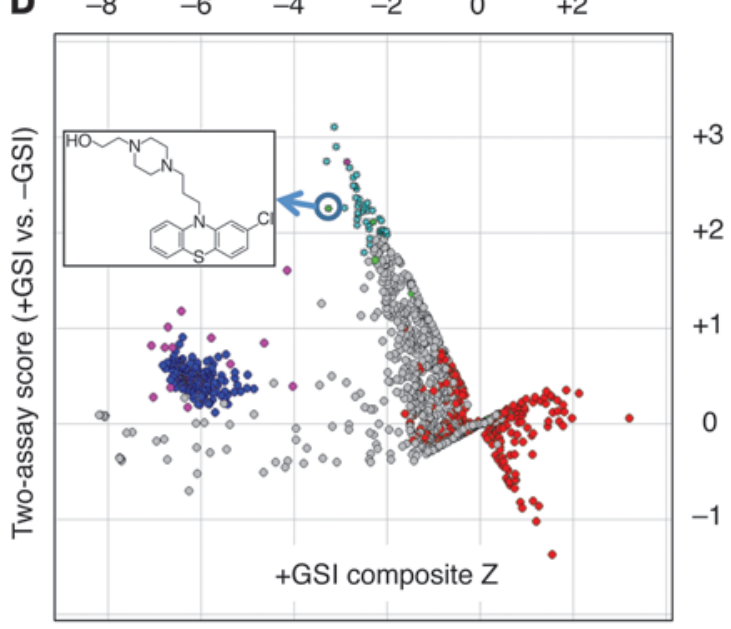

\section{Figure 2}

Results of a screen for small molecule inhibitors that synergize with the $\gamma$-secretase inhibitor compound $E$ to suppress the growth of the human T-ALL cell line KOPT-K1. KOPT-K1 cells were incubated in 384-well format with small molecules from the Broad Institute bioactives collection in the presence $(\mathbf{A})$ or absence $(\mathbf{B})$ of $\mathrm{GSI}$ (compound $\mathrm{E}, 0.1 \mu \mathrm{M})$ in duplicate. The effects of drugs on cell growth were assessed after 2 days by incubating with CellTiter-Blue (Promega)for an additional 4 hours, and each replicate compound measurement was converted to a $Z$ score relative to the corresponding DMSO-control distribution, as described for ChemBank (70). (C) ChemBank composite Z scores were computed to combine replicates within each condition (+GSI and-GSI), allowing the assays to be considered in a single plot; here, we sought compounds that were synergistic with GSI, i.e., they score with negative composite Z in the presence of GSI and have less effect in the absence of GSI. To express these preferences quantitatively, we devised a 2-assay score (D) that scales the +GSI composite Z both by the extent to which it points in the negative direction and by the extent to which it exceeds the -GSI composite Z (see Methods); 330 compounds tested met our initial criterion (scale factor > 0.5), of which 43 compounds, including PPZ (inset), were statistically distinguishable from the DMSO-control distribution. See Methods and Supplemental Figure 4 for additional details; screen data are available in ChemBank (see Methods).

Although we did not identify compounds that were selectively toxic to MYC-overexpressing thymocytes, multiple compounds with activity against both MYC-positive and -negative thymocytes were identified. Among these hits were numerous glucocorticoids, which are well known inducers of thymocyte apoptosis, as well as conventional chemotherapeutics that induce DNA damage (alkylating agents, topoisomerase inhibitors) (Figure $1 \mathrm{~B}$ and Supplemental Table 1). Many of these compounds are mainstays of contemporary T-ALL therapy, thus validating the utility of our approach. A number of novel compounds were also identified, including perphenazine (PPZ), an FDA-approved phenothiazine antipsychotic (Figure 1, B and C, and Supplemental Table 1). Secondary screening in zebrafish larvae $(n=6$ larvae per concentra- tion) confirmed that PPZ is active against MYC-overexpressing thymocytes in a dose-dependent manner (Figure 1D).

Human T-ALL screen for small molecules that synergize with $\gamma$-secretase inhibitors. In parallel, we conducted a cell-based screen with the Broad Institute bioactives chemical library collection (3,194 compounds) to identify drugs that synergistically suppress the growth of T-ALL cells in combination with gamma-secretase inhibitors (GSI). Roughly two-thirds of the small molecules in this collection $(n=2108)$ overlap with compounds screened in the zebrafish. The screen was conducted with KOPT-K1 cells, a NOTCH1-dependent T-ALL cell line that overexpresses the transcription factor TAL1, a feature associated with a relatively poor prognosis in this disease. We used the combination of GSI and dexamethasone, a known 

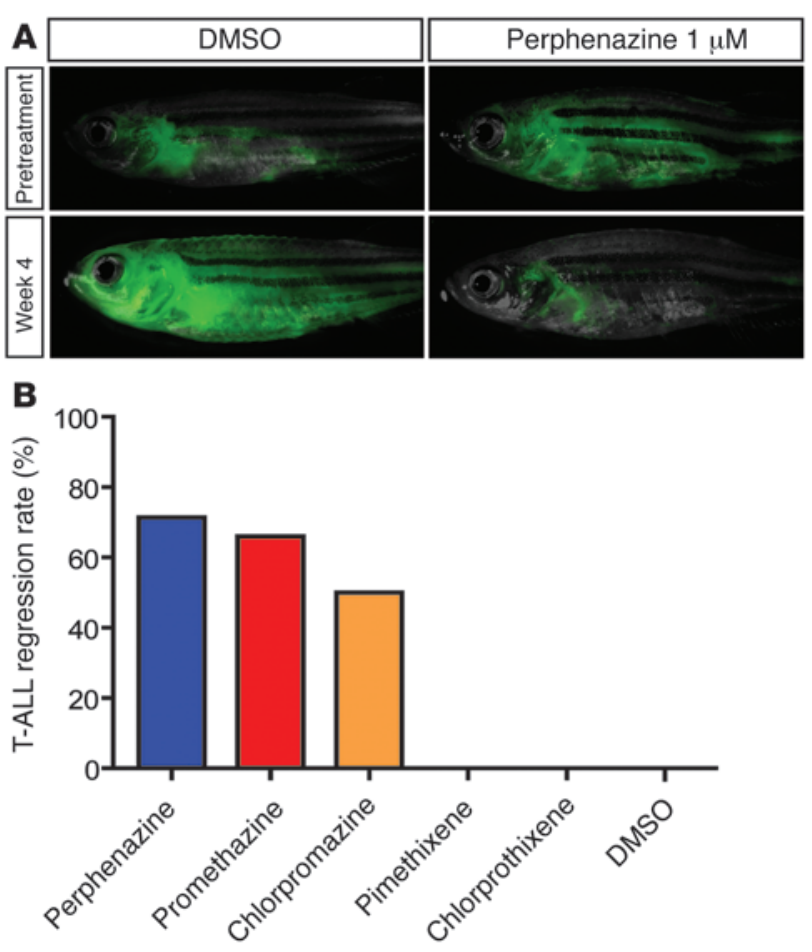

synergistic combination (25), as a positive control. We initially performed isobologram analysis to select concentrations of GSI (compound E, $100 \mathrm{nM}$ ) and dexamethasone (200 nM) that have a synergistic growth inhibitory effect on KOPT-K1 cells (Supplemental Figure 2) and then used these concentrations of drugs in screen optimization pilot studies. Under the final conditions selected, GSI versus GSI plus dexamethasone produced a Z score of 0.74 (Supplemental Figure 3); for point of reference, screens with $\mathrm{Z}$ scores greater than 0.5 are taken as having a high probability of detecting true positives.

We then screened the Broad Institute bioactives library in KOPT-K1 cells according to the strategy shown in Supplemental Figure 4. In brief, GSI/bioactive-compound interaction was assessed by independently scoring the effects of GSI plus each bioactive compound (Figure 2A) and the effects of each bioactive compound alone (Figure 2B). To identify compounds that synergize with GSI, we looked at the data using 2 different approaches. First, we combined the replicate data sets and plotted the composite $Z$ scores (a measure of difference from the control state) in the presence and absence of GSI (Figure 2C). We then scaled the composite $\mathrm{Z}$ score in the presence of GSI in a fashion that assessed each screened molecule for both the extent to which it suppressed T-ALL cell growth in the presence of GSI (the +GSI composite Z score) and the extent to which this suppression exceeded that observed in the absence of GSI (the so-called 2-assay score; Figure 2D). Using these metrics, the reproducible set of synergistic "hits" totaled 43 of 3,194 compounds screened, or $1.3 \%$ of compounds (for structure and identity of these compounds, see ChemBank [http://chembank.broadinstitute.org/ chemistry/search/execute.htm?id=5685551]). Notably, PPZ also scored as one of the top hits in the KOPT-K1 cell line screen.

Verification of the antileukemic activity of PPZ. Taken together, our 2 screens suggested that $\mathrm{PPZ}$ targets a conserved regulator of benign and malignant $\mathrm{T}$ cell progenitor growth or viability, and we there-

\section{Figure 3}

PPZ and a subset of related phenothiazines are active against established zebrafish T-ALL. (A) Representative images of zebrafish with established MYC-induced T-ALL treated with DMSO or PPZ $(1 \mu \mathrm{M})$. (B) Rate of T-ALL regression in zebrafish with established MYCinduced T-ALL, treated with $1 \mu \mathrm{M}$ of the indicated compound. A minimum of 8 zebrafish was analyzed per condition.

fore elected to study its effects on T-ALL cells in greater detail. We first tested PPZ in zebrafish with established tumors and found that PPZ is active against established MYC-induced T-ALL arising in a nonconditional rag2:EGFP-MYC transgenic zebrafish line (Figure 3, A and B), indicating that its activity is independent of 4-hydroxytamoxifen and the conditional MYC-ER fusion transgene used in the zebrafish-based screen. Activating Notch mutations are absent from tumors arising in this model (26); thus, drugs that score in this model do so through Notch-independent mechanisms. We also tested the activity of a series of structurally related phenothiazines and found that 2 other phenothiazines, promethazine and chlorpromazine, had antileukemic activity in zebrafish at $1 \mu \mathrm{M}$ concentration, whereas chlorprothixene and pimethixene lacked activity against established T-ALL in this model (Figure 3B).

We next extended studies with PPZ and other phenothiazines to mammalian T-ALL cell lines. PPZ as a single agent suppressed the growth of KOPT-K1 cells, the cell line used in the human T-ALL screen (Figure 4A), and the combination of PPZ and GSI caused moderately synergistic growth suppression of KOPT-K1 cells and a second human NOTCH1-dependent T-ALL cell line, DND41 (Figure 4B), as judged by isobologram analysis (Supplemental Table 2). Similar synergistic interactions between PPZ and GSI were observed in the notch1-dependent murine T-ALL cell lines 142 (Figure 4C and Supplemental Table 2) and 144 (Supplemental Table 2), both of which harbor a transgene driving expression of constitutively active kras as well as acquired activating mutations in notch1 (27). PPZ also suppressed the growth of SUPT13 (Figure 4D), a GSI-insensitive human T-ALL cell line that lacks NOTCH1 mutations, and PF382, a GSI-insensitive human T-ALL cell line that expresses a constitutively active mutated form of NOTCH1 (Supplemental Table 2). In these GSI-insensitive cell lines, little or no interaction between PPZ and GSI was observed in terms of cell numbers (Figure 4D) or isobologram analysis (Supplemental Table 2), suggesting that (as in zebrafish T-ALL) phenothiazines have antileukemic effects in mammalian T-ALL cell lines that are Notch-independent.

The mechanism of T-ALL growth suppression by PPZ was investigated in human cell lines and in zebrafish (Figure 5). PPZ treatment of human and murine T-ALL cell lines induced apoptosis, as assessed by the appearance of a sub-G1 DNA fraction (Figure 5, $\mathrm{A}$ and B), increased caspase activity (Figure 5, C and D), and increased annexin $V$ staining (Supplemental Figure 5A), effects that were enhanced by cotreatment with GSI (Figure 5, A, B, and D, and Supplemental Figure 5B). We also tested the activity of PPZ in established T-ALL tumors induced in rag2:EGFP-MYC;rag2:EGFP$B C L 2$ double-transgenic zebrafish (Figure 5E), and found that BCL2 overexpression completely blocked the activity of PPZ in all zebrafish tested $(n=9)$, confirming that PPZ acts via induction of mitochondrial apoptosis.

PPZ binds and activates the PP2A tumor suppressor. Phenothiazines are best known as inhibitors of dopaminergic signaling. Multiple dopamine receptors are expressed on $\mathrm{T}$ lineage cells and are proposed 
A
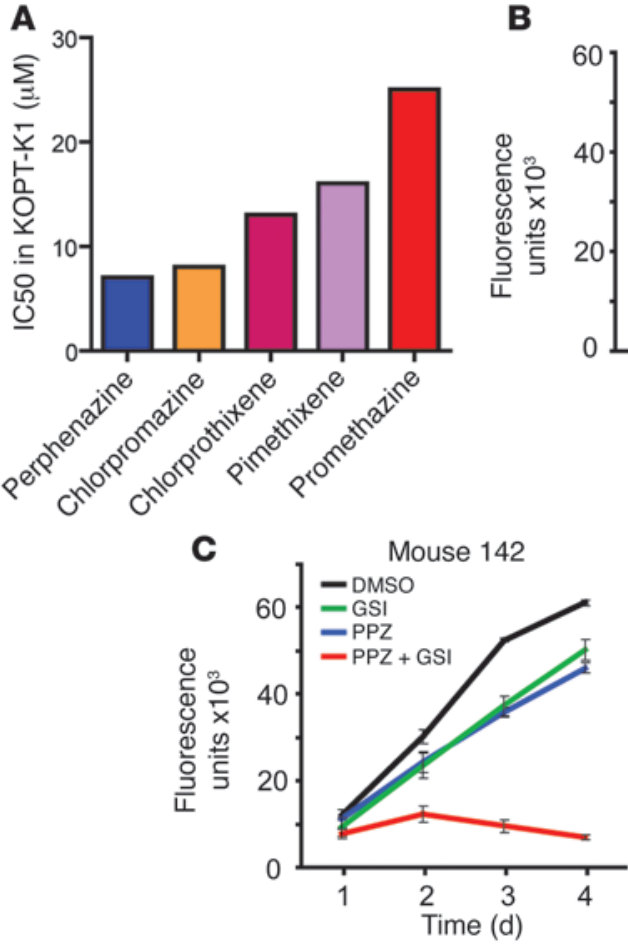

KOPT-K1

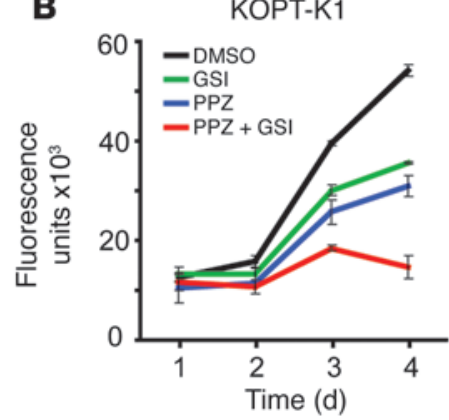

DND41

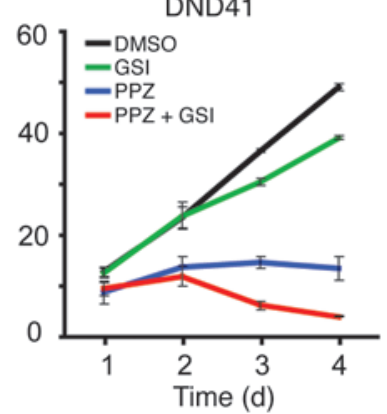

Figure 4

Activity of PPZ and related phenothiazines against mammalian T-ALL cell lines. (A) Growth suppression of KOPT-K1 cells by the indicated phenothiazines, expressed as IC 50 . (B-D) Effects of compound E (GSI) and PPZ on the growth of Notch1-dependent human KOPT-K1 and DND41 T-ALL cells (B), Notch1-dependent murine 142 T-ALL cells (C), and NOTCH1-independent human SUP-T13 T-ALL cells (D). In each experiment, each condition was assayed in triplicate, and all experiments were repeated at least twice. In B-D, PPZ and compound E were used at $10 \mu \mathrm{M}$ and $0.1 \mu \mathrm{M}$, respectively, while in (C) PPZ and compound E were used at $3 \mu \mathrm{M}$ and $6 \mathrm{nM}$, respectively.

to regulate immune function (28). However, the structurally unrelated dopamine inhibitors haloperidol, domperidone, and clozapine were not toxic to Myc-overexpressing thymocytes, had no effect on T-ALL progression in zebrafish, and did not affect human KOPT-K1 T-ALL cell growth (data not shown). Conversely, promethazine, a phenothiazine that does not inhibit dopaminergic signaling (29), decreased the growth of zebrafish T-ALLs and human KOPT-K1 T-ALL cells (Figure 3B and Figure 4A). Taken together, these data strongly suggest that inhibition of dopaminergic signaling is unrelated to the antileukemic activity of these compounds.

Another reported activity of phenothiazines that could abrogate mitogenic signaling is inhibition of calmodulin (30), which has broad roles in calcium-dependent intracellular signaling. However, we noted that W13, a relatively selective calmodulin inhibitor, is highly toxic to zebrafish larvae at low nanomolar concentrations (data not shown), presumably because calmodulin inhibition is not compatible with survival. Thus, while it is difficult to exclude some contribution of calmodulin inhibition to the observed effects of phenothiazines, calmodulin inhibition cannot explain the activity of phenothiazines in the zebrafish screen.

We were therefore left with the challenge of identifying the molecular target that was responsible for the activity of phenothiazines in our screens. To address this issue, we devised a quantitative mass spectrometry approach, termed activity correlation proteomics, that offers to improve the identification of biologically relevant targets of pleiotropic drugs. Briefly, we used fluoroustagged PPZ and fluorous ligand-affinity chromatography to pull- down protein targets from KOPT-K1 T-ALL cell lysates labeled with stable isotopes (SILAC) (31). These protein pull-downs were done in the presence or absence of competitor phenothiazines with varying $\mathrm{IC}_{50}$ for KOPT-K1 cell growth inhibition, and displacement of individual binding proteins from the fluorous PPZ matrix was assessed by quantitative mass spectrometry. We then rank ordered binding proteins based on the correlation between displacement by drug and the drug's $\mathrm{IC}_{50}$ in KOPT-K1 cells. This approach identified PPP2R1A as the protein whose binding affinity correlated most closely with the biologic activities of the phenothiazines tested (Figure 6, A and B). PPP2R1A is the A $\alpha$ scaffolding subunit of the protein phosphatase PP2A, and subsequent work confirmed that PPZ directly binds purified PP2A at low micromolar concentrations (Alex Kentsis and James E. Bradner, personal communication).

PP2A is a conserved heterotrimeric phosphatase that has many different isoforms, each comprising a scaffolding A subunit, a variable regulatory B subunit, and a catalytic $C$ subunit $(32,33)$. Best known as a tumor suppressor that is targeted by SV40 small $t$ antigen, PP2A is also a target of the oncogenic microRNA miR-19 (34), which collaborates with activated Notch1 to promote T-ALL development. The tumor-suppressive activity of PP2A is believed to be mediated through its ability to dephosphorylate diverse substrates implicated in oncogenic signaling, including AKT (35), p70S6K (36), MYC (37-39), ERK $(40,41)$, and $\operatorname{BAD}(42,43)$. Thus, if the antileukemic effects of PPZ and other phenothiazines in T-ALL are mediated through PP2A, then these drugs should stimulate 
A Human KOPT-K1 cells

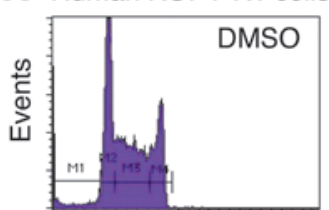

PI staining

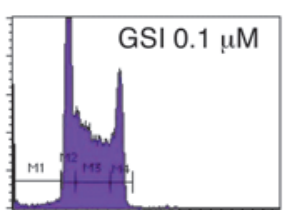

PI staining

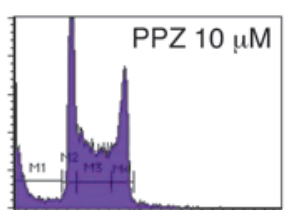

PI staining

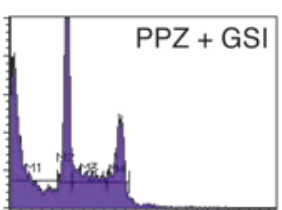

$\mathrm{PI}$ staining

B Mouse 142 cells

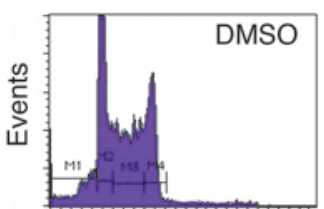

PI staining

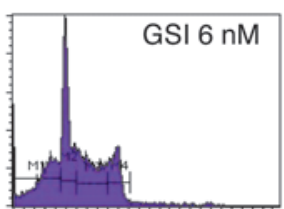

PI staining

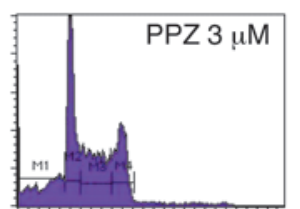

PI staining

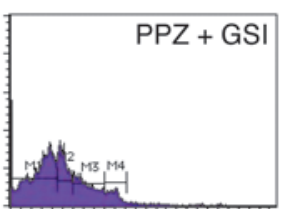

PI staining

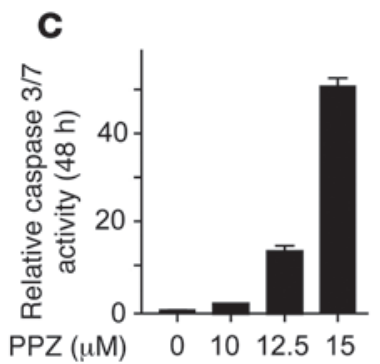

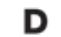

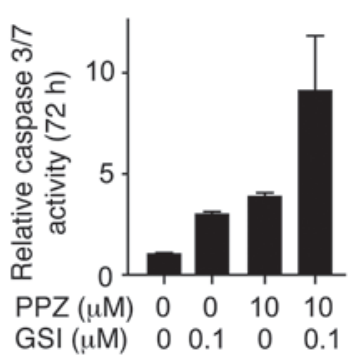

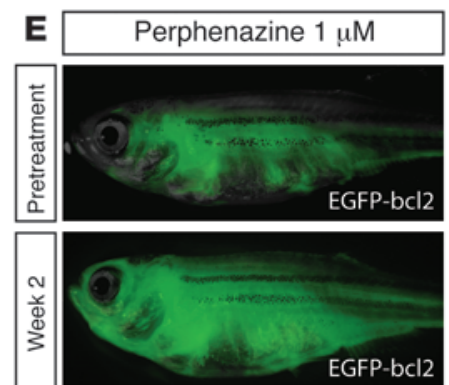

\section{Figure 5}

PPZ induces T-ALL cell apoptosis. (A and B) DNA histograms of human KOPT-K1 or mouse 142 T-ALL cells, respectively, treated with compound $\mathrm{E}$ (GSI) or PPZ alone and in combination for 96 hours. (C and D) PPZ increases caspase 3/7 activity in KOPT-K1 cells alone or in combination with GSI (compound $E$ ), used at the indicated concentrations for 48 hours. (E) A representative rag2:EGFP-MYC; rag2:EGFP-BCL2 double-transgenic zebrafish treated with $1 \mu \mathrm{M}$ PPZ is shown. PPZ had no activity in any of the $b c / 2$-transgenic zebrafish tested $(n=8)$.
PP2A activity. As predicted, incubation of KOPT-K1 cells with PPZ led to rapid dephosphorylation of the PP2A substrates AKT, ERK, p70S6K, MYC, and BAD (Figure 6, C-E), effects that were abrogated by addition of the phosphatase inhibitor okadaic acid (Figure 6D), an inhibitor of PP2A. Total AKT, ERK, p70S6K, and BAD protein levels were unaffected by PPZ, but the levels of MYC protein fell (Figure 6E), in line with prior reports showing that PP2A-mediated dephosphorylation of MYC residue 662 enhances MYC degradation (37). In addition, we noted that the effects of PPZ on PP2A substrates and growth of T-ALL cell lines such as KOPT-K1 were phenocopied by FTY720 (Fingolimod) (Supplemental Figure 6, A and B), an FDA-approved immunomodulatory drug previously shown to activate PP2A at low micromolar concentrations $(44,45)$. In contrast, the calmodulin inhibitor W13 had no effect on PP2A targets such as p70S6K (Supplemental Figure 6C).

To determine whether PP2A is required for the activity of PPZ, we first used shRNA to knock down the PP2A A $\alpha$ scaffolding subunit. PP2A A $\alpha$ knockdown impaired the PPZ-induced dephosphorylation of PP2A targets (Figure 7A) and also desensitized KOPT-K1 cells to the growth-suppressive effects of PPZ (Figure 8B). shRNA knock-down of a catalytic PP2A C $\alpha$ subunit had similar effects (Figure 7, C and D). Taken together, these data strongly suggest that growth-suppressive effects of PPZ on T-ALL cells are mediated through stimulation of PP2A activity.

$P P Z$ activates $P P 2 A$ and has antileukemic effects on primary buman $T$-ALL cells in vitro and in vivo. With these results in hand, it remained to be determined whether PPZ could inhibit the growth of primary T-ALL cells in vitro and in vivo, and if so, whether antileukemic effects were associated with dephosphorylation of PP2A targets. To address this issue in vitro, we tested a series of primary human T-ALLs (summarized in Supplemental Table 3) that had been expanded by passage through NSG mice for sensitivity to PPZ in a newly described serum-free culture system for T-ALL cells that relies on completely defined medium and feeder cells expressing the Notch ligand DLL-1 (46). We noted that low micromolar levels of PPZ resulted in dephosphorylation of multiple PP2A targets (AKT, p70S6K, and BAD) and impaired growth of all 4 T-ALLs tested under these conditions (Figure 8). Variable sensitivity to GSI and variable interaction between GSI and PPZ was also noted (Figure 8). We also tested an independent series of primary T-ALLs in a second culture system that relies on high levels of human serum and observed similar results, as all lines were growth inhibited by low micromolar concentrations of PPZ (Supplemental Figure 7).

One of the primary human T-ALL lines, hTALL2, grew sufficiently well in the serum-free culture system to permit luciferization with retrovirus, which facilitated drug testing in vivo. This was carried out in mice bearing calcium phosphate scaffolds seeded with human marrow-derived mesenchymal stem cells, an approach that allows for drug testing within a "humanized" microenvironment. Treatment of mice with PPZ $(10 \mathrm{mg} / \mathrm{kg}$ intraperitoneally daily) caused a significant decrease in growth of hTALL2 cells in the scaffolds (Figure 9A) and in murine spleen (Figure 9B). Importantly, a single dose of PPZ $(10 \mathrm{mg} / \mathrm{kg})$ also led to the dephosphorylation of the PP2A targets AKT, p70S6K, and BAD in hTALL2 blasts in vivo (Figure 9C). These results show that PPZ can produce antileukemic effects and stimulate PP2A activity in primary T-ALL lymphoblasts at doses that are tolerated by mice.

\section{Discussion}

NOTCH1 is a compelling therapeutic target in T-ALL, but the activity of GSIs as single agents in T-ALL preclinical models (47) and clinical trials (48) has been modest, suggesting the need to 
A

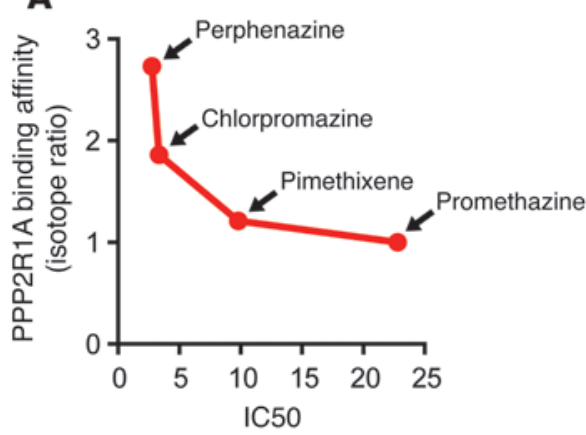

D

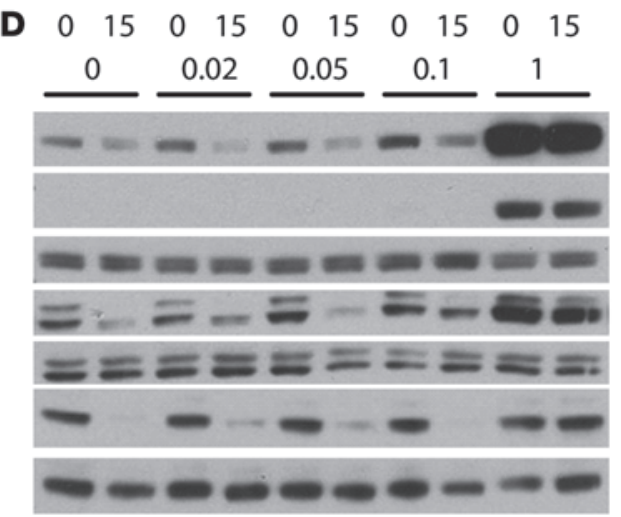

B

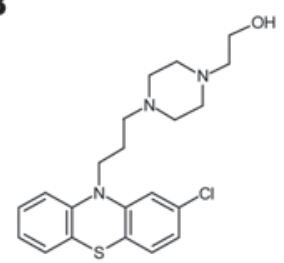

Perphenazine

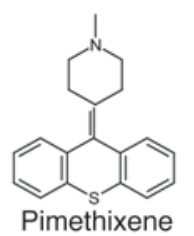

PPZ $(\mu \mathrm{M})$

$\mathrm{OA}(\mu \mathrm{M})$

$\mathrm{p}-\mathrm{AKT}(\mathrm{S} 473)$

pAKT (shorter exposure)

AKT

p-ERK

ERK

p-p70S6K(T389)

p70S6K

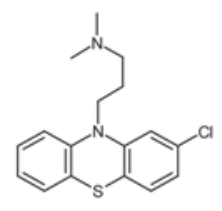

Chlorpromazine

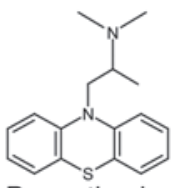

Promethazine
C $\frac{15 \min P P Z(\mu M)}{0 \quad 20 \quad 15 \quad 10}$

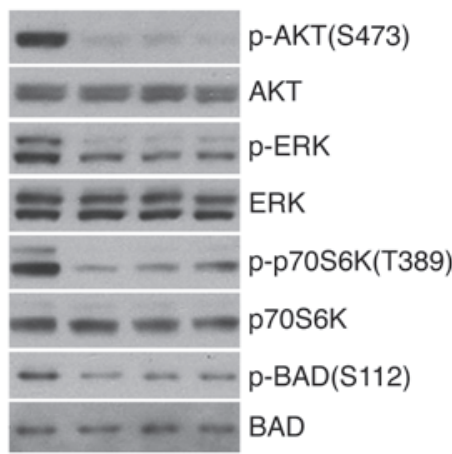

$\begin{array}{llllll}\text { E } & 0 & 20 & 15 & 10 & \mathrm{PPZ}(\mu \mathrm{M})\end{array}$

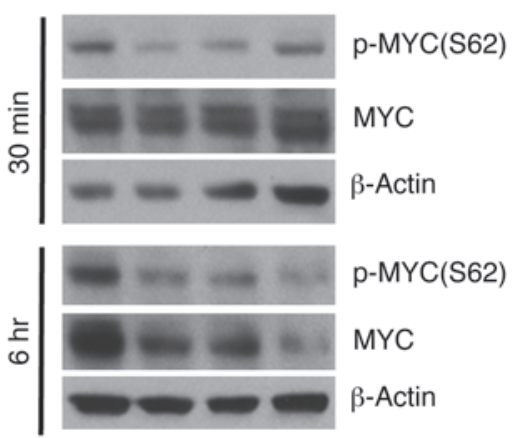

Figure 6

PPZ binds PP2A and leads to the rapid dephosphorylation of PP2A targets in KOPT-K1 T-ALL cells. (A) Activity correlation proteomics revealed that the biologic activity of phenothiazines correlates most closely to their binding affinity for PPP2R1A, the scaffolding A $\alpha$ subunit of the PP2A phosphatase, as described (Alex Kentsis and James E. Bradner, personal correspondence). (B) Structures of the phenothiazines tested. (C) Effects of PPZ on phosphorylation of AKT, ERK, p70S6K, and BAD. Lysates were prepared after 15 minutes of treatment. (D) The PP2A inhibitor okadaic acid (OA) antagonizes PPZ-mediated dephosphorylation of PP2A substrates. Lysates were prepared after 15 minutes of PPZ treatment. (E) Effects of PPZ on MYC phosphorylation and protein levels. Lysates were prepared after 30 minutes or 6 hours of PPZ treatment. In D, cells were pretreated with okadaic acid for 30 minutes prior to addition of PPZ.

combine Notch pathway inhibitors with other compounds with activity in this disease. We used complementary screens to discover small molecules with Notch-independent anti-T-ALL activity. Most small molecules scoring in both screens were glucocorticoids, providing further support for use of GSIs and glucocorticoids in combination in relapsed/refractory T-ALL (25). A previously unidentified hit emerging from the both screens was PPZ, which was shown to suppress the growth of piscine, murine, and human T-ALLs through induction of apoptosis. These effects of PPZ were linked by biochemical, cell biological, and genetic studies to activation of PP2A, a tumor-suppressive protein phosphatase. The ability of PPZ to activate PP2A thus represents the likely mechanism for the previously unexplained ability of phenothiazines to downregulate PI3K/AKT signaling and may explain the recurrent identification of these compounds in unbiased screens for agents with antineoplastic activity (18-21, 49-53).

Given that signaling via mutated KRAS (54) and receptors such as IL-7R $(55,56)$ and IGF-R1 (57) synergize with Notch signaling to induce and maintain T-ALL growth, combinations of agents that antagonize downstream effectors of these pathways are attractive therapeutic options in this disease. Indeed, prior work has shown that GSI in combination with TOR inhibitors $(9,47)$, PI3K inhibitors (58), and MAPK inhibitors (58) have increased activity over GSI alone against T-ALL. PP2A blunts signaling through multiple "nodes" downstream of surface receptors and RAS via dephosphorylation of signaling intermediates such as ERK $(40,41)$, AKT (35), and p70S6K (36) and can also inhibit growth by downregulating MYC (37-39) and promote apoptosis by dephosphorylating BAD $(42,43)$. In support of PPZ acting as a PP2A activator, our data show that PPZ treatment affects phosphorylation of each of these PP2A substrates, whereas shRNA knockdown of PP2A catalytic or scaffolding subunits impaired the antileukemic activity of PPZ. Withdrawal of Notch signaling via treatment with GSI or other inhibitors interferes with the growth of T-ALL cells through a number of mechanisms, including downregulation of MYC $(6,8,59)$, IL-7R (55, 60), and IGF-R1 (57), and suppression of PI3K/AKT signaling (61). The partially overlapping effects of PP2A stimulation and Notch withdrawal on key mitogenic signaling pathways would predict that drug combinations that hit both targets should have at least additive growth-suppressive effects on Notch-dependent T-ALL cells, consistent with our findings here with GSI and phenothiazines. 
A

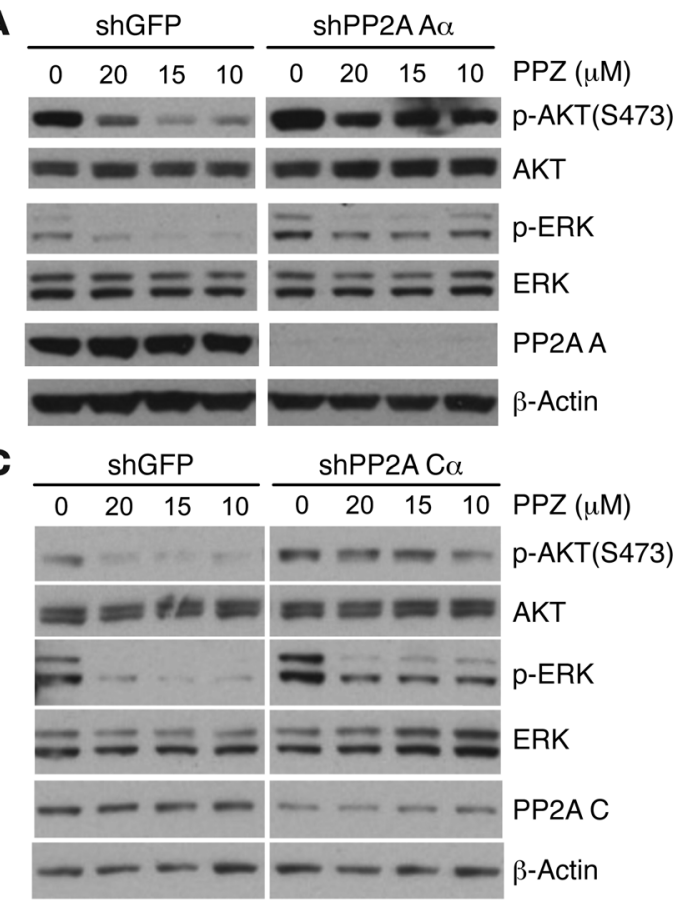

B
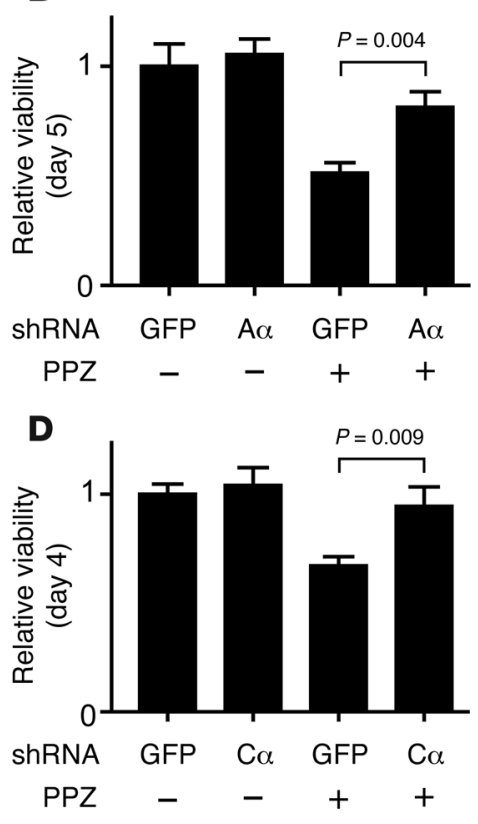

\section{Figure 7}

PP2A A $\alpha$ (structural) and $\mathrm{C} \alpha$ (catalytic) subunits are required for the growth-suppressive activity of PPZ in T-ALL cells. (A) Western blot analysis of KOPT-K1 T-ALL cells infected with shRNA specific for GFP (control) or PP2A $A \alpha$ and treated with the doses of PPZ shown. (B) Effect of the same shRNAs on KOPT-K1 T-ALL cell viability at day 5 of treatment with control or $5 \mu \mathrm{M} \mathrm{PPZ}$. (C) Western blot analysis of KOPT-K1 T-ALL cells infected with shRNA specific for GFP (control) or PP2A C $\alpha$ and treated with the doses of PPZ shown. (D) Effect of the same shRNAs on KOPT$\mathrm{K} 1$ cell viability after treatment with control (DMSO) or $7.5 \mu \mathrm{M} \mathrm{PPZ}$. In A and $\mathbf{C}$, cells were treated with $P P Z$ for 30 minutes.
Our data suggest that a combination of a GSI and a PP2A activator is an attractive strategy in relapsed/refractory T-ALL, one that would also merit consideration in other Notch-associated tumors, such as mantle cell lymphoma (62), chronic lymphocytic leukemia (63-65), and breast cancer (66), that have failed conventional therapies. A limitation is that PPZ and most other phenothiazines have marked extrapyramidal side effects, which is a limitation when considering these drugs as therapeutic agents in cancer, and have activities as antipsychotic drugs at low nanomolar concentrations, substantially below the levels that are needed to activate PP2A. It may be possible to alleviate these drawbacks by engineering phenothiazine derivatives that are more potent PP2A activators and yet lack activity on dopamine receptors; promethazine is an example of a phenothiazine with antileukemia activity that does not avidly bind dopamine receptors, suggesting that these 2 activities can be separated. An alternative approach is to use other PP2A activators with more favorable toxicity profiles that are already FDA approved, such as FTY720 (44). FTY720 is a potent inhibitor of the sphingosine-1-phosphate receptor that has activity in preclinical models of a variety of cancers, including chronic lymphocytic leukemia (67), chronic myelogenous leukemia blast crisis (45), B cell acute lymphoblastic leukemia (45), and mantle cell lymphoma (68). However, as with phenothiazines, the levels of FTY-720 that are required for PP2A stimulation are in the low micromolar range, making it a less than ideal clinical lead. It is likely that more active and selective PP2A-stimulating compounds will need to be developed if they are to have a robust clinical impact as anticancer drugs. Identification of the PP2A-stimulating effect of phenothiazines provides a new path forward for the development of small molecules with improved on-PP2A and diminished off-target activities.

\section{Methods}

Transgenic zebrafish lines. The rag2:MYC-ER; mitf, rag2:dsRed2, rag2:EGFP$M Y C$, and rag2:EGFP-BCL2 transgenic lines have previously been described $(12,23,69)$.
Zebrafish small molecule screen. At 3 dpf, transgenic zebrafish larvae expressing rag2:Myc-ER and rag2:dsRed2 transgenes (12) were arrayed into 96-well plates (3 larvae per well) and exposed to individual small molecules at doses of $12.5 \mu \mathrm{M}$ for small molecules and $1.25 \mu \mathrm{M}$ for bioactive lipids, together with $50 \mu \mathrm{g} / \mathrm{l}(129 \mathrm{nM})$ 4-hydroxytamoxifen to activate the Myc-ER transgene, as well as antibiotics to reduce bacterial overgrowth $(25$ units $/ \mathrm{ml}$ penicillin, $25 \mathrm{mg} / \mathrm{ml}$ streptomycin, and $5 \mathrm{mg} / \mathrm{ml}$ meropenem). Small molecules were from the following small molecule libraries: Lopac 1280 ( $n=1280$ compounds; Sigma-Aldrich); ICCB known bioactives $(n=480$ compounds; Enzo Life Sciences); the Prestwick Chemical Library ( $n=1200$ compounds; Prestwick Chemical); and the Spectrum Collection ( $n=2000$ compounds; MicroSource Discovery Systems). After 4 days of treatment, thymic fluorescence in individual larvae was assessed via fluorescence microscopy. Small molecules that induced general toxicity to any zebrafish larvae were discarded. Eight wells in each 96-well plate were dedicated to positive (dexamethasone $12.5 \mu \mathrm{M}$ ) and negative (DMSO) controls.

KOPT-K1 cell line screen. The screening method used for human T-ALL cells is shown schematically in Supplemental Figure 4. Briefly, KOPT-K1 cells (10,000 per well) were seeded using the MATRIX WellMate in 384-well plates in RPMI supplemented with $10 \%$ fetal calf serum, glutamine, and penicillin/streptomycin at $37^{\circ} \mathrm{C}$ with $5 \% \mathrm{CO}_{2}$. To assess interactions between GSI (compound E) and bioactive compounds, wells were then sequentially "pinned" with CyBi-Well in duplicate with compound E (100 nM; +GSI) or DMSO (-GSI) followed by a small molecule from the Broad Institute bioactives collection. Bioactive library compounds were delivered in a volume of $25 \mathrm{nl}$ to produce a final concentration that ranged from $5 \mu \mathrm{M}$ to $10 \mu \mathrm{M}$. Positive controls consisted of dexamethasone ( $200 \mathrm{nM})$ alone or dexamethasone ( $200 \mathrm{nM}$ ) plus GSI (100 $\mathrm{nM}$ compound E), while negative controls consisted of vehicle (DMSO) alone or GSI (100 nM compound E) alone. After 48 hours of treatment, CellTiter-Blue was added for 4 hours and viable cell numbers were determined by measuring fluorescence at $595 \mathrm{~nm}$ in a plate reader. High-throughput screening data were processed separately for the 2 assays (Figure 2A: +GSI; Figure 2B: -GSI) for deposition in ChemBank as described (70). Complete raw and processed data for these 


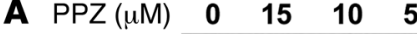

TTALL1

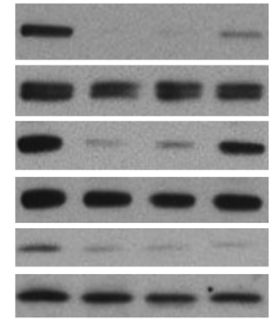

pAKT (S473)

AKT

p-p70S6K (T386)

p70S6K

$\mathrm{p}-\mathrm{BAD}(\mathrm{S} 112)$

$B A D$

B

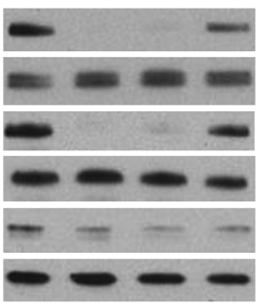

pAKT (S473)

AKT

p-p70S6K (T386)

hTALL2

p70S6K

p-BAD (S112)

BAD

C

$\begin{array}{lllll}P P Z(u M) & 0 & 15 & 10 & 5\end{array}$

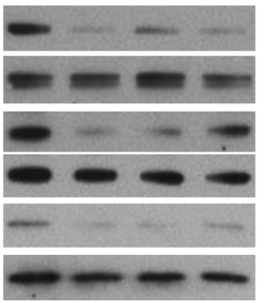

pAKT (S473)

AKT

p-p70S6K (T386)

hTALL6

p70s6K

p-BAD (S112)

BAD

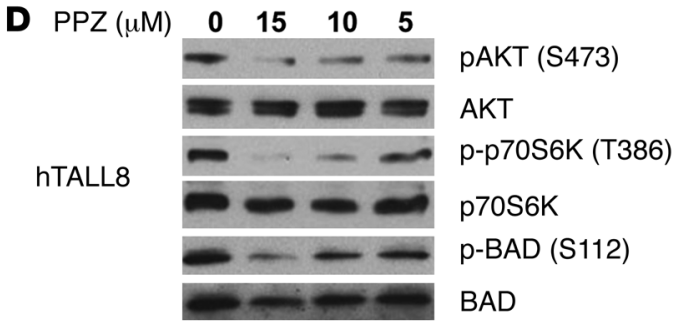

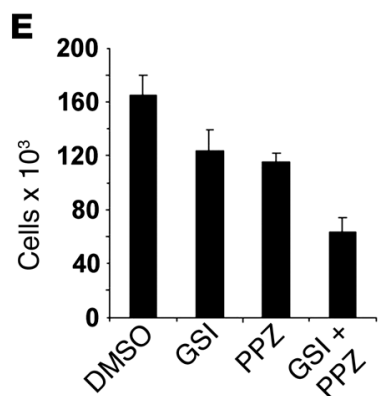
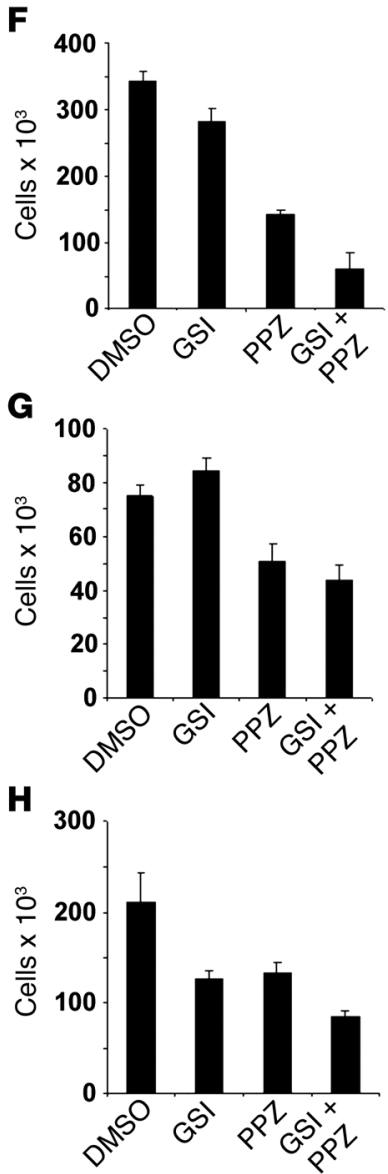

$-\left(Z_{+G S I}\right) \times F$, which clearly discriminates the compounds of interest when plotted against $Z_{+\mathrm{GSI}}$ (Figure 2D). Using an initial cutoff of $\mathrm{F}>0.5$, we identified 330 compounds, of which 43 compounds also were statistically distinct from the 2-assay DMSO distribution, modeled as a multivariate normal distribution, when subjected to Holm-Bonferroni multiple hypothesis test correction.

Cell culture. All cells were cultured at $37^{\circ} \mathrm{C}$ with $5 \% \mathrm{CO}_{2}$. Established cell lines were grown in RPMI supplemented with $10 \%$ fetal calf serum, glutamine, and penicillin/streptomycin. Primary human T-ALLs were grown in shortterm cultures on MS5-DLL1 stromal cells in either high-serum (71) or in serum-free defined conditions (46) as described.

Cell viability assays. Cell viability was measured using the CellTiter-Blue cell viability assay kit (Promega). Cells were plated in the black-walled 96-well plates and treated with chemical compounds. Then $20 \mu \mathrm{l}$ of CellTiter-Blue reagent was added to wells, each of which contained $100 \mu \mathrm{l}$ of cells. After 4 hours at $37^{\circ} \mathrm{C}$, fluorescence was recorded in a SpectraMax M3 microplate reader (Molecular Devices) to measure resazurin reduction.

Isobologram analysis. To determine whether drugs have synergistic antileukemia cell growth effects, cells were incubated with various concentrations of drugs for 48 hours and then incubated with CellTiter-Blue for an addition 4 hours. Cell numbers were determined by measuring fluorescence at $595 \mathrm{~nm}$ in a plate reader. Data were processed and subjected to isobologram analysis (72) using CalcuSyn to determine the combination index $(\mathrm{CI})$ at $\mathrm{ED}_{50}$. A CI of less than 0.7 at $\mathrm{ED}_{50}$ is taken to be indicative of a synergistic interaction.

Cell-cycle analysis. Cells were washed once with cold PBS and fixed with $90 \%$ ethanol overnight at $-20^{\circ} \mathrm{C}$. Cells were then washed once with cold PBS and resuspended in propidium iodide (PI) staining solution $(20 \mu \mathrm{g} / \mathrm{ml}$ PI, $200 \mu \mathrm{g} / \mathrm{ml}$ DNAse-free RNAse A, and $0.1 \%$ [v/v] Triton $\mathrm{X}-100$ in PBS). Following incubation at $37^{\circ} \mathrm{C}$ for 15 minutes, the samples were analyzed in a FACSCalibur flow cytometer (BD Biosciences) using Cell Quest software.

Figure 8

PPZ induces rapid dephosphorylation of PP2A targets and inhibits growth of cultured primary human T-ALL cells. (A-D) Western blots showing the effects of incubation for 15 minutes with the indicated doses of PPZ. (E-H) Effects of GSI (compound E, $1 \mu \mathrm{M})$ and PPZ $(5 \mu \mathrm{M})$ alone and in combination on cell growth relative to vehicle-treated control cells. Cells were grown in defined medium on MS5-DLL-1 feeder cells. Cell counts were determined after 3 days of treatment.

screening experiments are available via ChemBank (http://chembank. broadinstitute.org/assays/view-project.htm?id=1000654). To combine the results of the 2 assays quantitatively, we defined a trigonometric scaling function to express our preferences that (a) + GSI composite $Z$ scores be negative, and (b) + GSI composite $Z$ score amplitudes exceed -GSI composite $Z$ score amplitudes; such compounds should fall left of the DMSO-control distribution, but above the main diagonal in a plot of -GSI composite $Z$ versus + GSI composite $Z$ (Figure $2 \mathrm{C}$ ). Using the ratio of ChemBank composite $Z$ scores $(-\mathrm{GSI} /+\mathrm{GSI})$, we defined $\theta=\arctan \left(Z_{-\mathrm{GSI}} / Z_{+\mathrm{GSI}}\right)$ and expressed our scale factor as $F=\cos (-\theta) \cos (2 \theta)$, with the 2 factors respectively expressing our 2 preferences. As a 2 -assay composite score, we take
Apoptosis assays. Caspase 3 and 7 activities were measured using the CaspaseGlo 3/7 assay system (Promega). Following the treatment of KOPT-K1 cells with DMSO, GSI, PPZ, or GSI plus PPZ, 5,000 cells from each sample were plated in the white-walled 96-well plates with a final volume of $100 \mu \mathrm{l}$. An equal volume of Caspase-Glo $3 / 7$ reagent was then added to each well. After 1 hour at room temperature, luminescence was measured with a SpectraMax M3 microplate reader (Molecular Devices). As a second measure of apoptosis, cells were stained with annexin $\mathrm{V}$ and PI using Apoptosis Detection Kit I (BD Biosciences) per the manufacturer's instructions, and then analyzed in a FACSalibur flow cytometer (BD Biosciences) using Cell Quest software. 

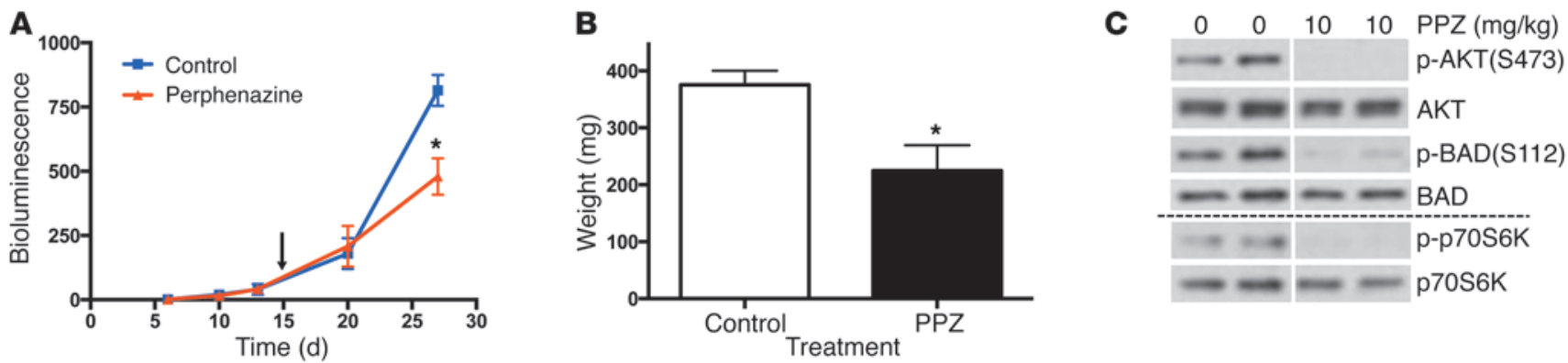

\section{Figure 9}

PPZ inhibits growth and dephosphorylates PP2A targets in primary human T-ALL cells in vivo. (A and B) Effects of treatment with PPZ $(10 \mathrm{mg} / \mathrm{kg} / \mathrm{d})$ on growth of human hTALL2 cells in NSG mice. (A) Effects on tumor cell growth were assessed by measuring the bioluminescence of luciferized hTALL2 cells in subcutaneous calcium phosphate scaffolds seeded with human mesenchymal stem cells. Arrow indicates the time of initiation of PPZ treatment. ${ }^{*} P<0.0182$. (B) Spleen weights of treatment and control mice at times of necropsy. ${ }^{*} P<0.05$. (C) Western blots prepared from hTALL2 cells harvested from engrafted mice 3 hours after a single dose of PPZ (10 mg/kg). Each lane corresponds to a different animal. The blot stained for phospho-p70S6K and total p70S6K was prepared from a second gel that was loaded with the same samples and run in parallel.

Murine xenograft assays. Retrovirus was made by transfecting MLCP, an MSCV-based retrovector encoding firefly luciferase, mCherry, and puromycin phosphotransferase, into Phoenix GP/293T cells (provided by David Williams, Boston Children's Hospital). Transduced human hTALL2 T-ALL cells were isolated based on mCherry expression using a FACSAria II SORP UV cell sorter (BD Biosciences). Luciferized hTALL2 cells were injected intrascaffold into NOD/SCID $\gamma$-chain knockout (NSG) mice bearing subcutaneous biphasic calcium phosphate scaffolds seeded with mesenchymal stem cells and followed for tumor development using bioluminescence imaging as described (73). To assess the antileukemic effects of PPZ, mice were treated daily with $10 \mathrm{mg} / \mathrm{kg}$ of PPZ by intraperitoneal injection and T-ALL growth was monitored by bioluminescence. To assess the effect of PPZ on phosphorylation of PP2A substrates in T-ALL cells in vivo, mice bearing bone scaffolds loaded with hTALL2 cells were treated with vehicle or a single dose of PPZ $(10 \mathrm{mg} / \mathrm{kg})$ intraperitoneally. Three hours later, cells were harvested from each scaffold and $2 \times 10^{7}$ cells were incubated with human CD45 MicroBeads (Miltenyi Biotec). Beads were subsequently washed once with cold PBS, $\mathrm{pH} 7.4$, containing $0.5 \%$ BSA and $2 \mathrm{mM}$ EDTA, and resuspended in $500 \mu \mathrm{l}$ of PBS. Bound cells were separated from beads on an MS column using a MACS Separator and immediately lysed for Western blot analyses.

Western blotting and antibodies. Cells were washed with cold PBS and resuspended in a lysis buffer (50 mM Tris, pH 8.0, 5 mM EDTA, $100 \mathrm{mM} \mathrm{NaF}$, $30 \mathrm{mM}$ pyrophosphate, $2 \mathrm{mM}$ molybdate, $2 \mathrm{mM}$ vanadate, $1 \% \mathrm{NP}-40$ ) supplemented with protease inhibitors (Sigma-Aldrich). Cell lysates were incubated on ice for 15 minutes and then centrifuged for 15 minutes at $12,000 \mathrm{~g}$ at $4^{\circ} \mathrm{C}$. Protein concentration was measured by Bradford assay (Bio-Rad), and equal amounts of protein were loaded on SDS-polyacrylamide gels and resolved by electrophoresis. Proteins were stained following transfer to PVDF membranes with the following specific antibodies: anti-pAKT(S473), anti-AKT, anti-pERK(T202/Y204), anti-ERK, anti-pP70S6K(S389), antiP70S6K, anti-pBAD(S112), anti-BAD, anti-MYC, and anti-PP2A subunit C (all from Cell Signaling Technology); pMYC(S62) (Abcam); and anti$\beta$-actin (Sigma-Aldrich). Staining was developed with Super Signal West Pico Chemiluminescent Substrate or Super Signal West Dura Extended Duration Substrate (Thermo Scientific) for 2 minutes at room temperature and documented by exposure to $x$-ray film (Kodak).

$P P 2 A$ shRNA knockdown. Knockdown of PP2A A $\alpha$ (encoded by $P P P 2 R 1 A)$ was performed using the pMKO.1-shAa vector as described (74). Knockdown of PP2A C $\alpha$ (encoded by PPP2CA) was performed with
pLKO.1-puro shPP2A C $\alpha$ obtained from Dana Farber Cancer Institute RNAi core facility. The targeting sequence inserted in pLKO.1-puro shPP2A is $5^{\prime}$-CCGGCCCATGTTGTTCTTTGTTATTCTCGAGAATAACAAAGAACAACATGGGTTTTT-3'. pLKO.1-puro-GFP was used as an shRNA control vector.

Chemicals. PPZ, chlorpromazine, chlorprothixene, pimethixene, promethazine, and domeridone were purchased from Sigma-Aldrich. Compound E and FTY720 were purchased from Enzo Life Sciences. Okadaic acid was purchased from LC Laboratories.

Statistics. Statistical significance of isobologram analyses was determined using Calcusyn, while differences in spleen weights were determined by $t$ test, performed with Prism software. Statistical analyses of KOPT-K1 cell line-screening data were performed as described in the Figure 2 legend and elsewhere in Methods.

Study approval. All animal studies were approved by the Dana Farber Cancer Institute Animal Care and Use Committee.

\section{Acknowledgments}

PP2A A $\alpha$ shRNA was a gift from W.C. Hahn. We wish to thank P. Ballerini, J. Landman-Parker, T. Leblanc, and A. Baruchel from Hôpital Trousseau and Hôpital R Debré for help in collection of patient samples and S. Blacklow for advice on technical and conceptual aspects of these studies. This work was supported by NIH grants 1K08CA133103 (to A. Gutierrez) and 5P01CA109901 (to A.T. Look); a Specialized Center of Research grant from the Leukemia and Lymphoma Society (to J.C. Aster and J.E. Bradner); grants from the William Lawrence Blanche Hughes Foundation (to A. Gutierrez, J.C. Aster, A.T. Look, and J.E. Bradner); a grant from the Bear Necessities Pediatric Cancer Foundation (to A. Gutierrez); and grants from Ligue Nationale contre le Cancer, Association Laurette Fugain, Institut National du Cancer (INCA), Universités Paris Diderot and Paris Sud, INSERM, CEA and Canceropole Ile de France (StemPole) (to F. Pflumio, S. Poglio, and B. Uzan). R. Groen has received funding from the People Programme (Marie Curie Actions) of the European Union's Seventh Framework Programme FP7/2007-2013/ under REA grant agreement no. 302428. A. Gutierrez is a research fellow of the Gabrielle's Angel Foundation for Cancer Research and is a previous scholar of the American Society of Hematology Harold Amos Medical Faculty Development Program. The T-ALL cell line screen was funded in part with federal funds from the NCI Initia- 
tive for Chemical Genetics under contract no. N01-CO-12400. The content of this publication does not necessarily reflect the views or policies of the Department of Health and Human Services, nor does mention of trade names, commercial products, or organizations imply endorsement by the US Government.

Received for publication October 1, 2013, and accepted October 30, 2013.

1. Marks DI, et al. T-cell acute lymphoblastic leukemia in adults: clinical features, immunophenotype, cytogenetics and outcome from the large randomised prospective trial (UKALL XII/ECOG 2993). Blood. 2009;114(25):5136-5145.

2. Goldberg JM, et al. Childhood T-cell acute lymphoblastic leukemia: the Dana-Farber Cancer Institute acute lymphoblastic leukemia consortium experience. J Clin Oncol. 2003;21(19):3616-3622.

3. Ko RH, et al. Outcome of patients treated for relapsed or refractory acute lymphoblastic leukemia: a Therapeutic Advances in Childhood Leukemia Consortium study. J Clin Oncol. 2010; 28(4):648-654

4. Koch U, Radtke F. Notch in T-ALL: new players in a complex disease. Trends Immunol. 2011;32(9):434-442.

5. Kopan R, Ilagan MX. The canonical Notch signaling pathway: unfolding the activation mechanism. Cell. 2009;137(2):216-233.

6. Weng AP, et al. c-Myc is an important direct target of Notch1 in T-cell acute lymphoblastic leukemia/ lymphoma. Genes Dev. 2006;20(15):2096-2109.

7. Sharma VM, et al. Notch1 contributes to mouse $\mathrm{T}$-cell leukemia by directly inducing the expression of c-myc. Mol Cell Biol. 2006;26(21):8022-8031.

8. Palomero T, et al. NOTCH1 directly regulates c-MYC and activates a feed-forward-loop transcriptional network promoting leukemic cell growth. Proc Natl Acad Sci US A. 2006;103(48):18261-18266.

9. Chan SM, Weng AP, Tibshirani R, Aster JC, Utz PJ. Notch signals positively regulate activity of the mTOR pathway in T-cell acute lymphoblastic leukemia. Blood. 2007;110(1):278-286.

10. Felsher DW, Bishop JM. Reversible tumorigenesis by MYC in hematopoietic lineages. Mol Cell. 1999; 4(2):199-207.

11. Langenau DM, et al. Myc-induced T cell leukemia in transgenic zebrafish. Science. 2003; 299(5608):887-890.

12. Gutierrez A, et al. Pten mediates Myc oncogene dependence in a conditional zebrafish model of $\mathrm{T}$ cell acute lymphoblastic leukemia. J Exp Med. 2011; 208(8):1595-1603

13. Weng AP, et al. Activating mutations of NOTCH1 in human $\mathrm{T}$ cell acute lymphoblastic leukemia. Science. 2004;306(5694):269-271.

14. Gutierrez A, et al. High frequency of PTEN, PI3K, and AKT abnormalities in T-cell acute lymphoblastic leukemia. Blood. 2009;114(3):647-650.

15. Palomero T, Ferrando A. Oncogenic NOTCH1 control of MYC and PI3K: challenges and opportunities for anti-NOTCH1 therapy in T-cell acute lymphoblastic leukemias and lymphomas. Clin Cancer Res. 2008;14(17):5314-5317.

16. Subramaniam PS, et al. Targeting nonclassical oncogenes for therapy in T-ALL. Cancer Cell. 2012; 21(4):459-472.

17. Zhu HG, Tayeh I, Israel L, Castagna M. Different susceptibility of lung cell lines to inhibitors of tumor promotion and inducers of differentiation. J Biol Regul Homeost Agents. 1991;5(2):52-58.

18. Kau TR, et al. A chemical genetic screen identifies inhibitors of regulated nuclear export of a Forkhead transcription factor in PTEN-deficient tumor cells. Cancer Cell. 2003;4(6):463-476.

19. Choi JH, et al. Potential inhibition of PDK1/Akt signaling by phenothiazines suppresses cancer cell
Address correspondence to: A. Thomas Look, Department of Pediatric Oncology, Dana-Farber Cancer Institute, 44 Binney St., Mayer 630, Boston, Massachusetts 02115, USA. Phone: 617.632.5826; Fax: 617.632.6989; E-mail: thomas_look@dfci.harvard.edu. Or to: Jon C. Aster, Department of Pathology, Brigham and Women's Hospital, 75 Francis St., Boston, Massachusetts 02115, USA. Phone: 617.525.4370; Fax: 617.525.4422; E-mail: jaster@rics.bwh. harvard.edu. proliferation and survival. Ann N Y Acad Sci. 2008; 1138:393-403.

20. Koren J, et al. Facilitating Akt clearance via manipulation of Hsp70 activity and levels.J Biol Chem. 2010; 285(4):2498-2505.

21. Rho SB, Kim BR, Kang S. A gene signature-based approach identifies thioridazine as an inhibitor of phosphatidylinositol-3'-kinase (PI3K)/AKT pathway in ovarian cancer cells. Gynecol Oncol. 2011; 120(1):121-127.

22. Sachlos E, et al. Identification of drugs including a dopamine receptor antagonist that selectively target cancer stem cells. Cell. 2012;149(6):1284-1297.

23. Langenau DM, Feng H, Berghmans S, Kanki JP, Kutok JL, Look AT. Cre/lox-regulated transgenic zebrafish model with conditional myc-induced T cell acute lymphoblastic leukemia. Proc Natl Acad SciU S A. 2005;102(17):6068-6073.

24. Lister JA, Robertson CP, Lepage T, Johnson SL, Raible DW. nacre encodes a zebrafish microphthalmia-related protein that regulates neuralcrest-derived pigment cell fate. Development. 1999; 126(17):3757-3767.

25. Real PJ, et al. Gamma-secretase inhibitors reverse glucocorticoid resistance in $\mathrm{T}$ cell acute lymphoblastic leukemia. Nat Med. 2009;15(1):50-58.

26. Blackburn JS, et al. Notch signaling expands a premalignant pool of T-cell acute lymphoblastic leukemia clones without affecting leukemia-propagating cell frequency. Leukemia. 2012;26(9):2069-2078.

27. Ashworth TD, et al. Deletion-based mechanisms of Notch1 activation in T-ALL: key roles for RAG recombinase and a conserved internal translational start site in Notch1. Blood. 2010;116(25):5455-5464.

28. Pacheco R, Prado CE, Barrientos MJ, Bernales S. Role of dopamine in the physiology of T-cells and dendritic cells. J Neuroimmunol. 2009;216(1-2):8-19.

29. Horn AS, Post ML, Kennard O. Dopamine receptor blockade and the neuroleptics, a crystallographic study. J Pharm Pharmacol. 1975;27(8):553-563.

30. Jaszczyszyn A, et al. Chemical structure of phenothiazines and their biological activity. Pharmacol Rep. 2012;64(1):16-23.

31. Ong SE, et al. Identifying the proteins to which small-molecule probes and drugs bind in cells. Proc Natl Acad Sci U S A. 2009;106(12):4617-4622.

32. Shi Y. Serine/threonine phosphatases: mechanism through structure. Cell. 2009;139(3):468-484.

33. Virshup DM, Shenolikar S. From promiscuity to precision: protein phosphatases get a makeover. Mol Cell. 2009;33(5):537-545.

34. Mavrakis KJ, et al. Genome-wide RNA-mediated interference screen identifies miR-19 targets in Notch-induced T-cell acute lymphoblastic leukaemia. Nat Cell Biol. 2010;12(4):372-379.

35. Sablina AA, Hector M, Colpaert N, Hahn WC. Identification of PP2A complexes and pathways involved in cell transformation. Cancer Res. 2010; 70(24):10474-10484.

36. Hahn K, Miranda M, Francis VA, Vendrell J, Zorzano A, Teleman AA. PP2A regulatory subunit PP2A-B' counteracts S6K phosphorylation. Cell Metab. 2010; 11(5):438-444

37. Yeh E, et al. A signalling pathway controlling c-Myc degradation that impacts oncogenic transformation of human cells. Nat Cell Biol. 2004;6(4):308-318

38. Arnold HK, Sears RC. Protein phosphatase 2A regulatory subunit B56alpha associates with c-myc and negatively regulates c-myc accumulation. $\mathrm{Mol}$ Cell Biol. 2006;26(7):2832-2844.

39. Junttila MR, et al. CIP2A inhibits PP2A in human malignancies. Cell. 2007;130(1):51-62.

40. Chen Z, et al. MAP kinases. Chem Rev. 2001; 101(8):2449-2476.

41. Ugi S, Imamura T, Ricketts W, Olefsky JM. Protein phosphatase $2 \mathrm{~A}$ forms a molecular complex with Shc and regulates She tyrosine phosphorylation and downstream mitogenic signaling. Mol Cell Biol. 2002;22(7):2375-2387.

42. Chiang CW, et al. Protein phosphatase 2A activates the proapoptotic function of $\mathrm{BAD}$ in interleukin-3-dependent lymphoid cells by a mechanism requiring 14-3-3 dissociation. Blood. 2001; 97(5):1289-1297.

43. Chiang CW, et al. Protein phosphatase 2A dephosphorylation of phosphoserine 112 plays the gatekeeper role for BAD-mediated apoptosis. Mol Cell Biol. 2003;23(18):6350-6362.

44. Matsuoka Y, Nagahara Y, Ikekita M, Shinomiya T. A novel immunosuppressive agent FTY720 induced Akt dephosphorylation in leukemia cells. Br J Pharmacol. 2003;138(7):1303-1312.

45. Neviani P, et al. FTY720, a new alternative for treating blast crisis chronic myelogenous leukemia and Philadelphia chromosome-positive acute lymphocytic leukemia. JClin Invest. 2007;117(9):2408-2421.

46. Yost AJ, et al. Defined, serum-free conditions for in vitro culture of primary human T-ALL blasts. Lenkemia. 2013;27(6):1437-1440.

47. Cullion K, et al. Targeting the Notch 1 and mTOR pathways in a mouse T-ALL model. Blood. 2009; 113(24):6172-6181

48. DeAngelo DJ, Stone RM, Silverman LB, Aster JC. A phase I clinical trial of the Notch inhibitor MK-0752 in patients with T-cell acute lymphoblastic leukemia/lymphoma (T-ALL) and other leukemias. J Clin Oncol. 2006;24:6585.

49. Sachlos E, et al. Identification of drugs including a dopamine receptor antagonist that selectively target cancer stem cells. Cell. 2012;149(6):1284-1297.

50. Zhelev Z, et al. Phenothiazines suppress proliferation and induce apoptosis in cultured leukemic cells without any influence on the viability of normal lymphocytes. Phenothiazines and leukemia. Cancer Chemother Pharmacol. 2004;53(3):267-275.

51. Gil-Ad I, Shtaif B, Levkovitz Y, Dayag M, Zeldich E, Weizman A. Characterization of phenothiazineinduced apoptosis in neuroblastoma and glioma cell lines: clinical relevance and possible application for brain-derived tumors. J Mol Neurosci. 2004; 22(3):189-198.

52. Gil-Ad I, et al. Phenothiazines induce apoptosis in a B16 mouse melanoma cell line and attenuate in vivo melanoma tumor growth. Oncol Rep. 2006; 15(1):107-112.

53. Sangodkar J, et al. Targeting the FOXO1/KLF6 axis regulates EGFR signaling and treatment response. J Clin Invest. 2012;122(7):2637-2651.

54. Chiang MY, et al. Leukemia-associated NOTCH1 alleles are weak tumor initiators but accelerate K-ras-initiated leukemia. J Clin Invest. 2008; 118(9):3181-3194.

55. Gonzalez-Garcia S, et al. CSL-MAML-dependent Notch 1 signaling controls $\mathrm{T}$ lineage-specific 
IL-7R\{alpha\} gene expression in early human thymopoiesis and leukemia. J Exp Med. 2009; 206(4):779-791.

56. Zenatti PP, et al. Oncogenic IL7R gain-of-function mutations in childhood T-cell acute lymphoblastic leukemia. Nat Genet. 2011;43(10):932-939.

57. Medyouf $\mathrm{H}$, et al. High-level IGF1R expression is required for leukemia-initiating cell activity in T-ALL and is supported by Notch signaling. J Exp Med. 2011;208(9):1809-1822.

58. Dail M, et al. Mutant Ikzf1, KrasG12D, and Notch1 cooperate in $\mathrm{T}$ lineage leukemogenesis and modulate responses to targeted agents. Proc Natl Acad Sci US A. 2010;107(11):5106-5111.

59. Subramanyam D, Krishna S. c-Myc substitutes for Notch1-CBF1 functions in cooperative transformation with papillomavirus oncogenes. Virology. 2006;347(1):191-198

60. Wang $\mathrm{H}$, et al. Genome-wide analysis reveals conserved and divergent features of Notch $1 /$ RBPJ binding in human and murine $\mathrm{T}$ lymphoblastic leukemia cells. Proc Natl Acad Sci U S A. 2011; 108(36):14908-14913.
61. Palomero T, et al. Mutational loss of PTEN induces resistance to NOTCH1 inhibition in T-cell leukemia. Nat Med. 2007;13(10):1203-1210.

62. Kridel R, et al. Whole transcriptome sequencing reveals recurrent NOTCH1 mutations in mantle cell lymphoma. Blood. 2012;119(9):1963-1971.

63. Di Ianni M, et al. A new genetic lesion in B-CLL: NOTCH1 PEST domain mutation. Br J Haematol. 2009;146(6):689-691.

64. Fabbri G, et al. Analysis of the chronic lymphocytic leukemia coding genome: role of NOTCH1 mutational activation. J Exp Med. 2011;208(7):1389-1401.

65 . Puente XS, et al. Whole-genome sequencing identifies recurrent mutations in chronic lymphocytic leukaemia. Nature. 2011;475(7354):101-105.

66 . Robinson DR, et al. Functionally recurrent rearrangements of the MAST kinase and Notch gene families in breast cancer. Nat Med. 2011; 17(12):1646-1651.

67. Liu Q, et al. FTY720 demonstrates promising preclinical activity for chronic lymphocytic leukemia and lymphoblastic leukemia/lymphoma. Blood. 2008;111(1):275-284
68. Liu Q, et al. FTY720 shows promising in vitro and in vivo preclinical activity by downmodulating Cyclin D1 and phospho-Akt in mantle cell lymphoma. Clin Cancer Res. 2010;16(12):3182-3192.

69. Langenau DM, et al. Suppression of apoptosis by bcl-2 overexpression in lymphoid cells of transgenic zebrafish. Blood. 2005;105(8):3278-3285.

70. Seiler KP, et al. ChemBank: a small-molecule screening and cheminformatics resource database. Nucleic Acids Res. 2008;36(Database issue):D351-359.

71. Armstrong F, et al. NOTCH is a key regulator of human T-cell acute leukemia initiating cell activity. Blood. 2009;113(8):1730-1740.

72. Tallarida RJ. Drug synergism: its detection and applications. J Pharmacol Exp Ther. 2001; 298(3):865-872.

73. Groen RW, et al. Reconstructing the human hematopoietic niche in immunodeficient mice: opportunities for studying primary multiple myeloma. Blood. 2012;120(3):e9-e16.

74. Sablina AA, et al. The tumor suppressor PP2A Abeta regulates the RalA GTPase. Cell. 2007; 129(5):969-982. 